PALMA VM, PINTO TMP, MACIEL RM, DANESI CC, FERRAZZO KL. Reação liquenoide oral ao amálgama: uma lesão ainda vista. Rev. Odontol. Univ. Cid. São Paulo 2018 jan-mar; 30(1): 95-102

ISSN 1983-5183

\title{
REAÇÃO LIQUENOIDE ORAL AO AMÁLGAMA: UMA LESÃO AINDA VISTA
}

\author{
Victor de Mello PALMA ${ }^{1}$ \\ victorpalmaod@hotmail.com
}

Tatiana Militz Perrone PINTO²

tatimilitz@hotmail.com

Roberto Marinho MACIEL ${ }^{3}$

marinhomacielr@gmail.com

Cristiane Cademartori DANESI ${ }^{4}$

cristianedanesi@gmail.com

Kivia Linhares FERRAZZO5

kivialinhares@uol.com.br

\section{RESUMO}

O amálgama dental ainda é amplamente utilizado na prática odontológica, apesar do acordo assinado na Convenção de Minamata, na qual 140 países comprometeram-se a reduzir o uso do mercúrio. O uso desse material restaurador pode desencadear reações liquenoides orais (RLO) cujas lesões apresentam semelhanças clínicas e histológicas com líquen plano oral (LPO), dificultando o diagnóstico. Aqui relatamos o caso de uma paciente do sexo feminino, de 72 anos, que foi encaminhada com uma lesão na mucosa bucal esquerda, com 10 meses de evolução e queixa de dor. A lesão era adjacente ao segundo molar inferior esquerdo que tinha uma restauração de amálgama. A paciente não apresentava doença sistêmica ou hábitos deletérios e não estava usando drogas continuamente. O diagnóstico clínico presuntivo foi RLO versus LPO. Sessenta dias após a substituição da restauração do amálgama, observou-se a regressão completa da lesão e o diagnóstico final de RLO foi realizado. A avaliação clínica associada aos resultados obtidos após a substituição do material suspeito pode ser suficiente para estabelecer o diagnóstico, embora em alguns casos seja necessária a avaliação histopatológica.

PALAVRAS-CHAVE: AMÁLGAMA DENTÁRIO; LÍQUEN PLANO BUCAL; ERUPÇÕES LIQUENOIDES

\footnotetext{
Mestrando do Programa de Pós-Graduação em Ciências Odontológicas (PPGCO) - Universidade Federal de Santa Maria (UFSM)

Mestre em Ciências Odontológicas. Docente da Faculdade de Odontologia do Centro Universitário Franciscano (UNIFRA)

Docente no departamento de Patologia da Universidade Federal de Santa Maria (UFSM)

4 Doutora em Genética e Toxicologia Aplicada. Docente da Faculdade de Odontologia e do Programa de Pós-Graduação em Ciências Odontológicas da Universidade Federal de Santa Maria (UFSM)

5 Doutora em Patologia Bucal. Docente da Faculdade de Odontologia da Universidade Federal de Santa Maria (UFSM)
} 


\begin{abstract}
Dental amalgam is still widely used in dental practice, despite the agreement signed at the Minamata Convention, in which 140 countries have committed to reducing the use of mercury. The use of this restorative material may trigger oral lichenoid reactions (OLR) whose lesions show clinical and histological similarities with oral lichen planus (OLP), making diagnosis difficult. Here we report the case of a female patient, 72 -year-old, who was referred with a lesion in the left buccal mucosa, with 10 months of evolution and complaint of pain. The lesion was adjacent to the second lower left molar which had an amalgam restoration. The patient had no systemic disease or deleterious habits and was not using drugs continuously. The presumptive clinical diagnosis were OLR versus OLP. Sixty days after the replacement of amalgam restoration the complete regression of the lesion was observed and the final diagnosis of OLR was done. Clinical assessment associated with the results obtained after the replacement of suspect material may be sufficient to establish the diagnosis, although in some cases it may be necessary histopathological evaluation.
\end{abstract}

KEY WORDS: DENTAL AMALGAM; LICHEN PLANUS, ORAL; LICHENOID ERUPTIONS

\title{
INTRODUÇÃO
}

Em 2013, cerca de 140 países assinaram um acordo global que previa a redução, controle e eliminação de produtos contendo mercúrio. Nesse documento, o uso do amálgama dentário não foi banido, mas medidas que desencorajam e desfavorecem seu uso foram estimuladas. Apesar disso, em alguns países, 0 amálgama dental ainda é rotineiramente utilizado'.

O uso de materiais contendo mercúrio como o amálgama dentário pode desencadear reações liquenoides na mucosa oral ${ }^{2}$. As reações liquenoides orais (RLO) associadas ao amálgama não são lesões comuns e apresentam semelhanças clínicas e histológicas com líquen plano oral (LPO). No entanto, essas lesões mostram etiologia, tratamento e prognóstico distintos ${ }^{3,4}$.

Clinicamente, essas lesões podem apresentar diferentes aspectos clínicos, como estrias, placas, áreas eritematosas, lesões erosivas, atróficas ou ulceradas e aparência histológica bastante semelhante à do líquen plano oral ${ }^{3}$. Isso dificulta o diagnóstico dessas lesões. Além disso, RLOs têm sido consideradas por alguns autores como lesões com potencial de malignidade ${ }^{5}$.

Este artigo apresenta um caso de RLO relacionada a uma restauração de amálgama antiga em que, após a sua remoção, houve regressão completa da lesão dentro de sessenta dias e o diagnóstico final de RLO foi realizado.

\section{RELATO DE CASO}

Paciente do sexo feminino, de 72 anos, procurou atendimento odontológico queixando-se de uma lesão sintomática na mucosa oral no lado esquerdo, com dez meses de evolução. A paciente relatou ter sensação de queimação no local da lesão, especialmente com alimentos ácidos.

Durante a anamnese, ela relatou que, por orientação de um cirurgião-dentista, utilizou um corticosteroide tópico (triamcinolona) na lesão por 30 dias, o que levou à desaceleração do crescimento e 
PALMA VM, PINTO TMP, MACIEL RM, DANESI CC, FERRAZZO KL. Reação liquenoide oral ao amálgama: uma lesão ainda vista. Rev. Odontol. Univ. Cid. São Paulo 2018 jan-mar; 30(1): 95-102

ISSN 1983-5183

diminuição da sintomatologia. Após a descontinuação do fármaco, observou-se a recorrência da lesão.

O exame físico intraoral revelou uma lesão na mucosa bucal esquerda, com $1 \mathrm{~cm}$ de diâmetro, com aspecto eritroplásico, mostrando bordas esbranquiçadas e área atrófica central (Fig. 1). Houve uma forte associação clínica entre a lesão e uma restauração de amálgama grande e antiga presente no segundo molar inferior esquerdo, que parecia estar em processo de corrosão e oxidação. A paciente não apresentava doença sistêmica ou hábitos deletérios e não fazia o uso de drogas de uso contínuo.

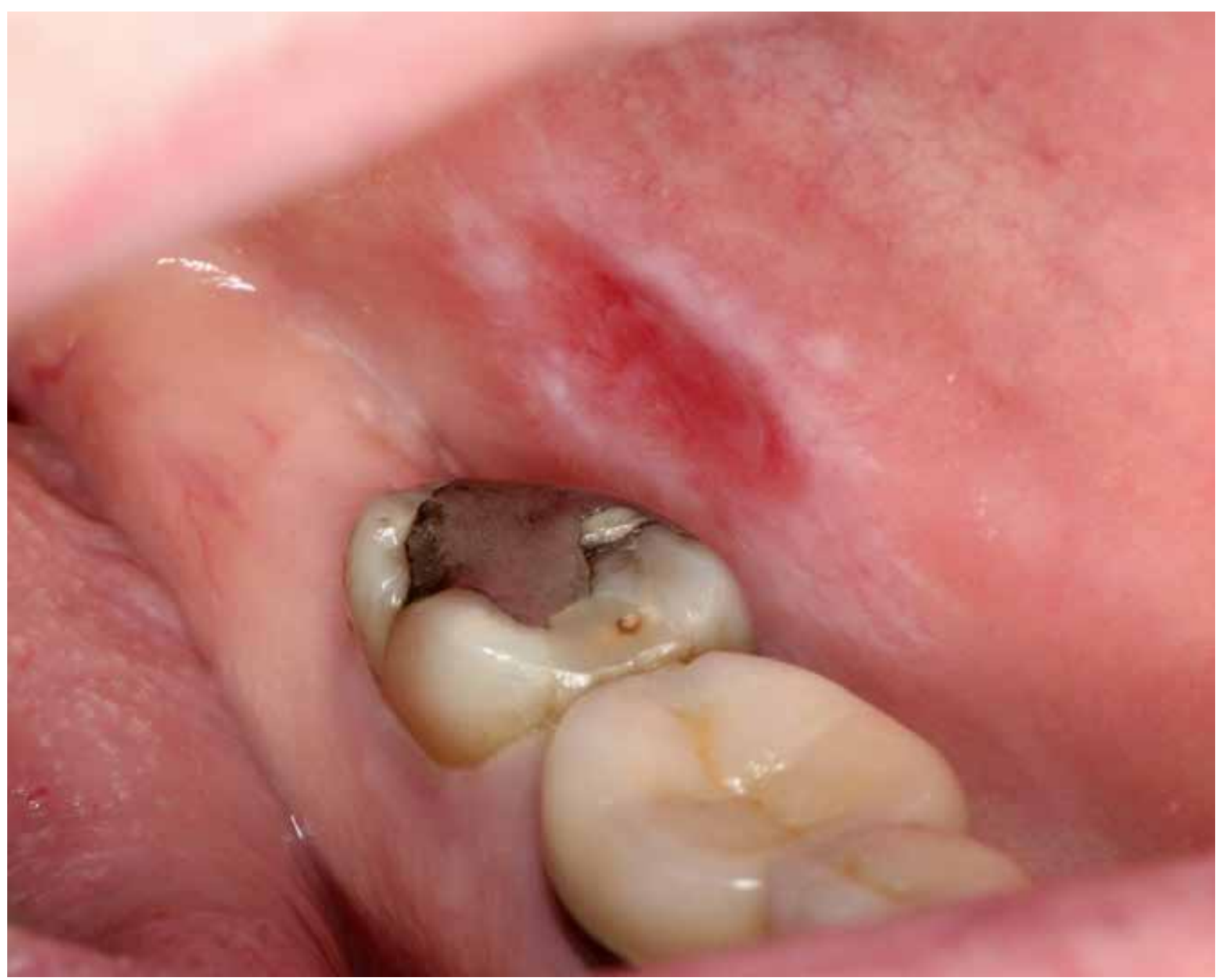

Figura 1 - Características clínicas iniciais: a lesão apresenta aspecto eritroplásico e atrófico central com bordas esbranquiçadas. Observe-se a forte associação clínica entre a lesão e uma restauração de amálgama grande e antiga no segundo molar inferior esquerdo.

Dados o relato e o aspecto clínico da lesão, as hipóteses diagnósticas foram de reação líquenoide oral ao amálgama versus líquen plano oral erosivo. $O$ tratamento inicialmente proposto consistiu em substituir a restauração de amálgama por outro material restaurador temporário (cimento de ionômero de vidro) e acompanhamento da paciente. Após 15 dias, houve uma boa melhora no aspecto clínico da lesão e redução da sintomatologia (Fig. 2). 
PALMA VM, PINTO TMP, MACIEL RM, DANESI CC, FERRAZZO KL. Reação liquenoide oral ao amálgama: uma lesão ainda vista. Rev. Odontol. Univ. Cid. São Paulo 2018 jan-mar; 30(1): 95-102

ISSN 1983-5183

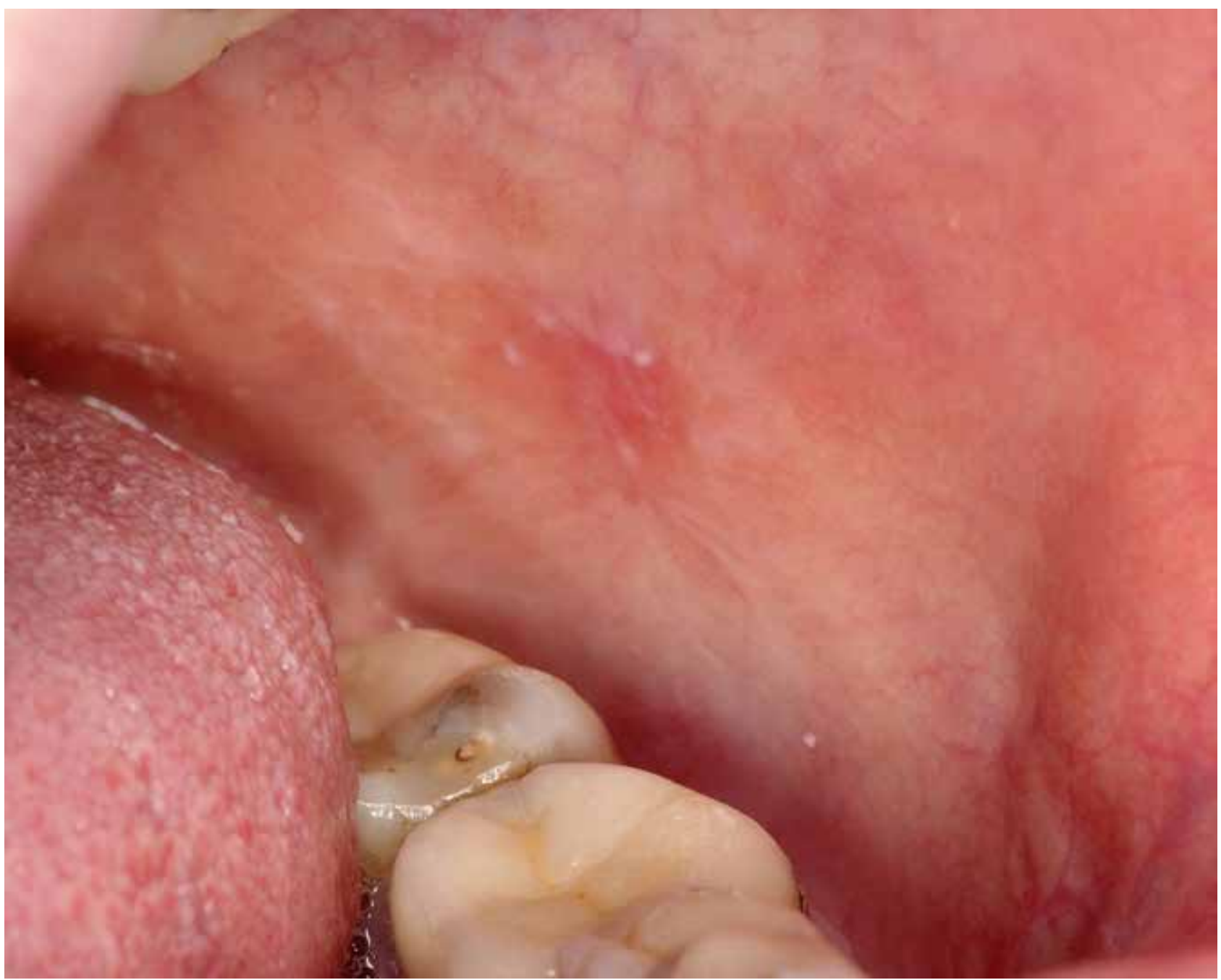

Figura 2 - Aspecto clínico 15 dias após a substituição do material restaurador: houve uma boa melhora no aspecto da lesão.

Dentro de 60 dias, observamos a regressão completa da lesão (Fig. 3), o que nos levou ao diagnóstico final de reação liquenoide oral ao amálgama. A paciente foi acompanhada periodicamente após regressão completa da lesão e não houve alteração no aspecto clínico da região, que manteve o aspecto da mucosa normal. 
PALMA VM, PINTO TMP, MACIEL RM, DANESI CC, FERRAZZO KL. Reação liquenoide oral ao amálgama: uma lesão ainda vista. Rev. Odontol. Univ. Cid. São Paulo 2018 jan-mar; 30(1): 95-102

ISSN 1983-5183

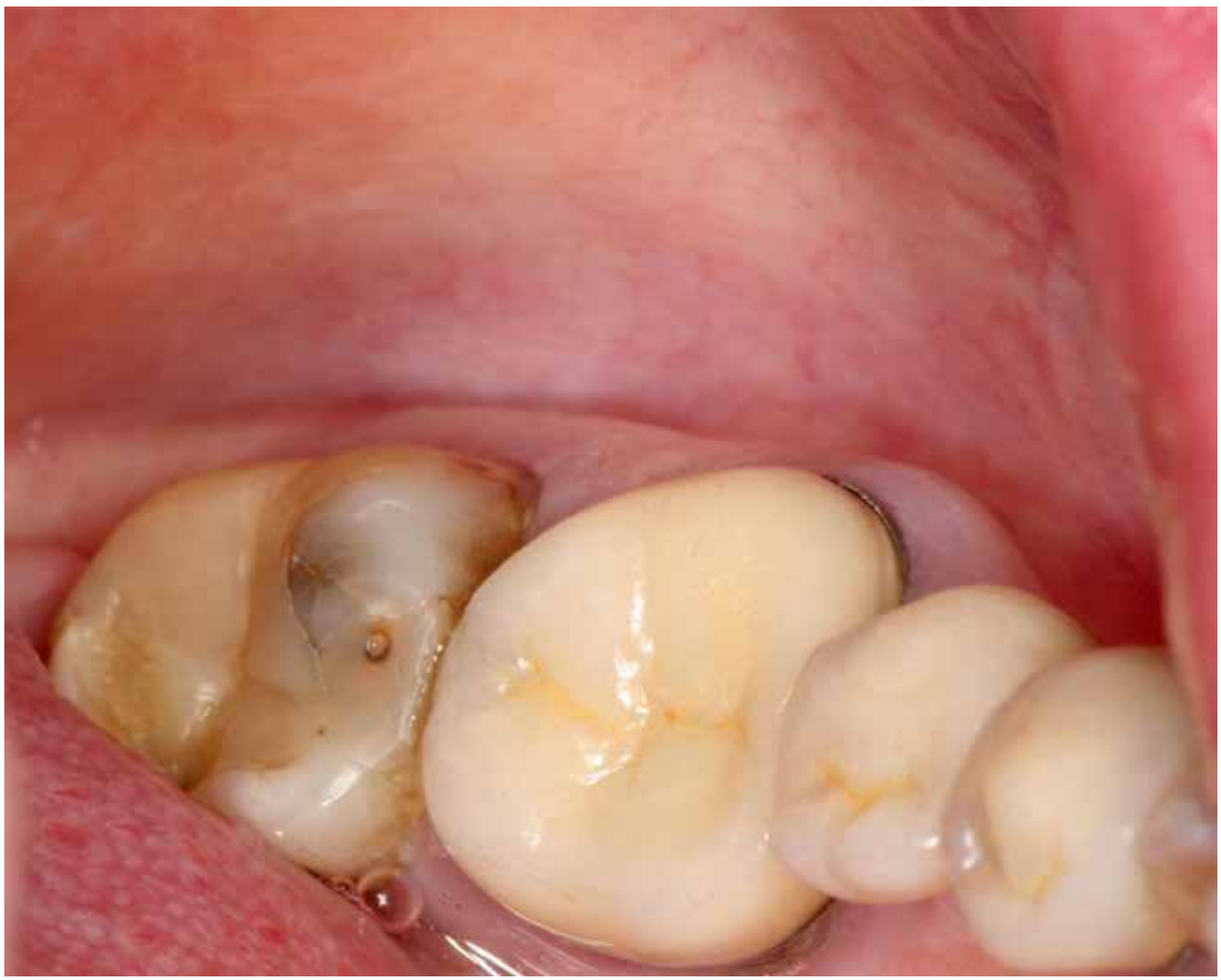

Figura 3 - Aspecto clínico 60 dias após a substituição do material restaurador: o desaparecimento completo da lesão pode ser observado.

Ao acompanhamento de 1 ano, a paciente relatou que estava passando por problemas familiares e forte estresse psicológico. 0 exame intraoral revelou uma pequena área eritematosa ao lado da região anteriormente afetada. Foi realizada uma biópsia incisional e os achados histológicos revelaram epitélio estratificado pavimentoso com atrofia focal sem displasia e, subjacente ao epitélio, um mínimo infiltrado inflamatório um mínimo não liquenoide. Em 15 dias, a mucosa novamente mostrou-se com aspecto de normalidade. A Tabela 1 resume os comportamentos que foram tomados com a paciente, com base na melhor evidência da literatura, como sugerido por Brignardello-Petersen ${ }^{6}$ (2015). 
PALMA VM, PINTO TMP, MACIEL RM, DANESI CC, FERRAZZO KL. Reação liquenoide oral ao amálgama: uma lesão ainda vista. Rev. Odontol. Univ. Cid. São Paulo 2018 jan-mar; 30(1): 95-102

ISSN 1983-5183

Tabela 1. Reação Liquenoide Oral (RLO). Questões clínicas, tipo de evidência e decisões clínicas.

\begin{tabular}{|c|c|c|}
\hline Questões Clínicas & Tipo de estudo/ Evidências & Decisão clínica \\
\hline $\begin{array}{l}\text { O amálgama dental pode } \\
\text { causar reações liquenoi- } \\
\text { des orais (RLO)? }\end{array}$ & $\begin{array}{l}\text { Estudo de caso controle. } 20 \text { pacientes com RLO } \\
\text { foram avaliados através dos níveis salivares de } \\
\text { interleucina } 6 \text { e } 8 \text { antes e após a remoção de } \\
\text { amálgama. O estudo mostrou que as restaurações } \\
\text { de amálgama podem causar RLO oral7. } \\
\text { Revisão sistemática. Há evidências de causalidade } \\
\text { entre a proximidade física da lesão e a restauração } \\
\text { do amálgama }{ }^{8} \text {. }\end{array}$ & $\begin{array}{l}\text { Como a lesão tinha proximida- } \\
\text { de física com a restauração de } \\
\text { amálgama, era possível prever } \\
\text { que a mesma fosse causada pelo } \\
\text { amálgama. }\end{array}$ \\
\hline $\begin{array}{l}\text { O exame histopatológico } \\
\text { é essencial para o diag- } \\
\text { nóstico de RLO relaciona- } \\
\text { do ao amálgama? }\end{array}$ & $\begin{array}{l}\text { Estudo observacional. Em 2006, um estudo tentou } \\
\text { determinar aspectos histológicos para diferenciar } \\
\text { o líquen plano oral das reações liquenoides orais } \\
\text { clinicamente diagnosticadas. Segundo os autores, } \\
\text { a histologia não parece ser útil }{ }^{4} \text {. }\end{array}$ & $\begin{array}{l}\text { A lesão foi diretamente associa- } \\
\text { da à restauração de amálgama } \\
\text { e apresentou forte associação } \\
\text { topográfica com o material } \\
\text { restaurador. Isso, combinado } \\
\text { com dados da literatura, levou } \\
\text { ao diagnóstico clínico de RLO ao } \\
\text { amálgama. Portanto, a biópsia } \\
\text { não foi realizada naquele mo- } \\
\text { mento. }\end{array}$ \\
\hline $\begin{array}{l}\text { A substituição da restau- } \\
\text { ração dental é indicada e } \\
\text { eficiente para promover a } \\
\text { regressão da RLO? }\end{array}$ & $\begin{array}{l}\text { Revisão sistemática. Issa et al. (2004) revisaram } \\
19 \text { estudos e descobriram que a maioria das RLOs } \\
\text { regrediu com a substituição do amálgama por } \\
\text { outros materiais restauradores }{ }^{8} \text {. } \\
\text { Estudo observacional. De acordo com Marell et } \\
\text { al. (2014), há evidências de que RLO em contato } \\
\text { físico próximo com amálgama pode regredir após } \\
\text { a substituição do material restaurador }{ }^{9} \text {. }\end{array}$ & $\begin{array}{l}\text { Substituição do amálgama } \\
\text { dentário por outros materiais } \\
\text { restauradores. }\end{array}$ \\
\hline $\begin{array}{l}\text { Existe risco de transforma- } \\
\text { ção maligna associada à } \\
\text { RLO? }\end{array}$ & $\begin{array}{l}\text { Revisão sistemática. Os autores pesquisaram na } \\
\text { literatura a prevalência de transformação maligna } \\
\text { da RLO. Os autores excluíram todos os trabalhos } \\
\text { em que os pacientes apresentavam displasia na } \\
\text { biópsia inicial. Apenas um estudo se encaixou } \\
\text { nesse critério. O risco pareceu maior em RLO, } \\
\text { seguido de LPO. A taxa de transformação maligna } \\
\text { da RLO foi de 3,2\% (10). }\end{array}$ & $\begin{array}{l}\text { Acompanhamento do paciente } \\
\text { por } 12 \text { meses. A lesão desapa- } \\
\text { receu dentro de } 60 \text { dias. Após } \\
12 \text { meses, uma pequena área } \\
\text { eritematosa foi vista. Dado o } \\
\text { risco de transformação maligna, } \\
\text { realizou-se uma biópsia para } \\
\text { excluir as alterações displásicas. }\end{array}$ \\
\hline
\end{tabular}

\section{DISCUSSÃO}

Atualmente, o uso consciente das melhores evidências científicas é essencial para tomar decisões clínicas sobre os diagnósticos e cuidados de pacientes individuais ${ }^{6}$. Durante a prática clínica, o cirurgiãodentista, às vezes, é confrontado com lesões na mucosa oral de difícil diagnóstico.

O diagnóstico de RLO é frequentemente clínico, não sendo necessária a biópsia, desde que ocorra o desaparecimento da lesão após a substituição do amálgama por outro material restaurador não metálico ${ }^{11}$. Na maioria, as RLOs regridem com a substituição do amálgama por outro material restaurador, enquanto nenhum caso de LPO foi resolvido após a substituição do material restaurador ${ }^{9}$ Nesse sentido, uma forte associação entre RLO e restauração de amálgama parece ser um bom critério para determinar com 
PALMA VM, PINTO TMP, MACIEL RM, DANESI CC, FERRAZZO KL. Reação liquenoide oral ao amálgama: uma lesão ainda vista. Rev. Odontol. Univ. Cid. São Paulo 2018 jan-mar; 30(1): 95-102

ISSN 1983-5183

segurança se o amálgama deve ou não deve ser substituídoª apesar de alguns autores declararem que a relação topográfica entre uma RLO e uma restauração de amálgama é útil, mas não é um critério conclusivo ${ }^{8}$.

O tratamento da RLO depende do agente etiológico. Conforme mencionado anteriormente ${ }^{9}$, a substituição do amálgama por outro material restaurador geralmente promove a regressão das lesões clinicamente relacionadas ao amálgama. No entanto, essa regressão da lesão pode levar vários meses e, se não houver resposta após a substituição do amálgama, uma biópsia deve ser realizada1". No caso relatado aqui, como houve uma regressão completa da lesão nos 60 dias após a substituição do amálgama, a biópsia não foi realizada naquele momento. No entanto, enfatizamos que, em certos casos, quando não há desaparecimento da lesão ou involução parcial, a avaliação histopatológica é necessária para confirmar ou descartar o diagnóstico clínico, como sugerido por outros autores ${ }^{11}$.

Apesar da pouca evidência científica, as RLOs são consideradas lesões potencialmente malignas ${ }^{11}$. Em apenas um estudo que adotou como critério exclusão de casos com displasia epitelial na biópsia inicial e diferenciou RLO de LPO, uma taxa de transformação maligna de 3,2\% de RLO foi encontrada, contra uma taxa de 1,09\% em LPO 5,10. A literatura apresenta alguns mecanismos que tentam explicar a fisiopatologia do potencial maligno do LPO. A explicação mais amplamente aceita é que as células inflamatórias associadas ao estresse oxidativo induzem a liberação de várias citocinas que influenciam o crescimento, a proliferação e a diferenciação das células, favorecendo assim a transformação maligna ${ }^{10}$. Levando em consideração que o componente inflamatório também está presente em RLO, inferimos que esse mecanismo também pode ser aplicado a essas lesões.

Portanto, a literatura sugere que pacientes com RLO devem ser monitorados duas vezes por ano, mesmo aqueles que não apresentam os fatores típicos de alto risco para o desenvolvimento de carcinoma epidermóide oral, a fim de se fazer um diagnóstico precoce de uma possível transformação maligna dessas lesões ${ }^{5,10}$. Parece seguro e prudente aconselhar os pacientes que possuem RLO causada por amálgama para substituirem as restaurações de amálgama por outros materiais restauradores que, além de eliminarem os sintomas, reduzem as chances de se desenvolver carcinoma epidermóide oral.

\section{CONCLUSÃO}

Apesar dos indicadores que apontam para a crescente interrupção do uso de materiais restauradores à base de mercúrio, o amálgama ainda é empregado na prática odontológica e, por vezes, pode gerar efeitos deletérios sobre a mucosa oral. 
ISSN 1983-5183

\section{REFERÊNCIAS}

1. BAKHURJI E, Scott T, Mangione T, Sohn W. Dentists' perspective about dental amalgam: current use and future direction. J Public Health Dent. 2017 Jun;77(3):207-15.

2. LIND PO, Hurlen B, Lyberg T, Aas E. Amalgam-related oral lichenoid reaction. Scand J Dent Res 1986 Oct;94(5):448-51.

3. VAN Der Meij EH, Van Der Waal I. Lack of clinicopathologic correlation in the diagnosis of oral lichen planus based on the presently available diagnostic criteria and suggestions for modifications. J Oral Pathol Med. 2003 Oct;32(9):507-12.

4. THORNHILL MH, Sankar V, Xu XJ, Barrett AW, High AS, Odell EW, et al. The role of histopathological characteristics in distinguishing amalgam-associated oral lichenoid reactions and oral lichen planus. J Oral Pathol Med. 2006 Apr;35(4):233-40.

5. VAN Der Meij EH, Mast H, Van Der Waal I. The possible premalignant character of oral lichen planus and oral lichenoid lesions: a prospective five-year follow-up study of 192 patients. Oral oncology 2007 Sep;43(8):742-8.

6. BRIGNARDELLO-PETERSEN R, Carrasco-Labra A, Glick M, Guyatt GH, Azarpazhooh A. A practical approach to evidence-based dentistry: $V$ : how to appraise and use an article about diagnosis. J Am Dent Assoc 2015 Mar;146(3):184-91.e1.

7. PEZELJ-RIBARIC S, Prpic J, Miletic I, Brumini G, Soskic MS, Anic I. Association between oral lichenoid reactions and amalgam restorations. J Eur Acad Dermatol Venereol 2008 Nov;22(10):1163-7.

8. ISSA Y, Brunton PA, Glenny AM, Duxbury AJ. Healing of oral lichenoid lesions after replacing amalgam restorations: a systematic review. Oral surgery, oral medicine, oral pathology, oral radiology, and endodontics Oral Surg Oral Med Oral Pathol Oral Radiol Endod 2004 Nov;98(5):553-65.

9. MARELL L, Tillberg A, Widman L, Bergdahl J, Berglund A. Regression of oral lichenoid lesions after replacement of dental restorations. J Oral Rehabil. 2014 May;41(5):381-91.

10. FITZPATRICK SG, Hirsch SA, Gordon SC. The malignant transformation of oral lichen planus and oral lichenoid lesions: a systematic review. J Am Dent Assoc. 2014 Jan;145(1):45-56.

11. VAN Der Waal I. Oral lichen planus and oral lichenoid lesions; a critical appraisal with emphasis on the diagnostic aspects. Med Oral Patol Oral Cir Bucal 2009 Jul 1;14(7):E3104.

RECEBIDO EM 17/10/2017

ACEITO EM 23/10,2017 